\title{
DIÂMETRO FOLICULAR E TAXA DE PRENHEZ EM PROTOCOLO DE SINCRONIZAÇÃO COM GNRH OU BENZOATO DE ESTRADIOL NO DIA $O$ EM VACAS DE CORTE
}

\author{
Ana Paula da Silveira ${ }^{1}$, Marina de Castro Martins ${ }^{2}$, Luiz Roberto Almeida Gabriel Filho ${ }^{3}$, Caliê Castilho ${ }^{4}$ \\ ${ }^{1}$ Mestre programa Mestrado Ciência Animal da UNOESTE \\ ${ }^{2}$ Aluna Medicina Veterinária - Presidente Prudente \\ ${ }^{3}$ Docente da UNESP - Campus Tupã - SP \\ ${ }^{4}$ Docente da Medicina Veterinária e Mestrado em Ciência Animal da UNOESTE
}

\section{RESUMO}

Neste trabalho objetivou-se avaliar a taxa de prenhez e o diâmetro folicular utilizando benzoato de estradiol (BE) ou hormônio liberador de gonadotrofinas $(\mathrm{GnRH})$ no dia da colocação do implante de progesterona (D0) em vacas de corte em lactação. Dois grupos foram testados em 2 experimentos. No Exp. 1 foram utilizadas 61 vacas da raça Nelore divididas em 2 grupos: G-BE $(n=32)$ e $G-G n R H(n=29)$. No D0 foi colocado implante de P4 (CIDR ${ }^{\circledR}$ ) e aplicados $2 \mathrm{~mL}$ de BE (G-BE) ou 2,5 mL de GnRH (G-GnRH). No D9 foi realizada ultrassonografia (US) para medir o diâmetro do folículo dominante (FD) presente no ovário e retirado o implante, concomitante à administração de $2,5 \mathrm{~mL}$ de PGF2 $\alpha$ e $0,25 \mathrm{~mL}$ de cipionato de estradiol (E.C.P. ${ }^{\circledR}$ ) seguida de remoção dos bezerros. Após 48 horas todas as vacas foram inseminadas e os bezerros retornaram às mães. No Exp. 2 foram utilizadas 50 vacas seguindo o mesmo protocolo descrito acima, porém foi avaliada a prenhez sem realização de US ovariana. Não houve diferença $(p>0,05)$ na taxa de prenhez entre os tratamentos, BE $(55 \%)$ ou GnRH $(41 \%)$, mas o diâmetro folicular foi significativamente maior $(p<0,05)$ nas vacas prenhes tratadas com $B E(10,7 \mathrm{~mm}$ vs $8,5 \mathrm{~mm})$ e nas vacas tratadas com $\mathrm{GnRH}$ não houve diferença $(p>0,05)$ entre as prenhes e não prenhes $(11,6 \mathrm{~mm}$ vs $10,2 \mathrm{~mm})$. Concluí-se que o uso de GnRH no Do não melhora a taxa de prenhez em vacas no pó-parto e o diâmetro folicular foi maior $(p<0,05)$ nas vacas prenhes quando comparado as não prenhes apenas no G-BE.

Palavras-chave: IATF; pós-parto; progesterona; vacas zebuínas.

\section{FOLLICLE DIAMETER AND PREGNANCY RATE IN SYNCHRONIZATION PROTOCOLS WITH GNRH OR ESTRADIOL BENZOATE ON DAY O IN BEEF COWS}

\begin{abstract}
This work aimed to evaluate the pregnancy rate and follicular diameter using EB or GnRH on the insertion of progesterone implant (D0) in lactating beef cows. Two groups were tested in two experiments. In Exp. 1 were used 61 Nelore cows divided into two groups: G-BE $(n=32)$ and $G-G n R H(n=29)$, on D0 was inserted P4 implant (CIDR囚) and applied $2 \mathrm{~mL}$ of $\mathrm{BE}$ (G-BE) or $2.5 \mathrm{~mL} \mathrm{GnRH}(\mathrm{G}-\mathrm{GnRH})$. In D9 was performed ultrasonography (U.S.) to measure the diameter of the dominant follicle (DF) present in the ovary and the implant was removed, with concomitant administration of $2.5 \mathrm{~mL}$ of PGF2 $\alpha$ and estradiol cypionate (ECPß) followed by calves removal. After 48 hours all the cows were inseminated and the calves returned. In Exp. 2 50 cows were used following the same protocol described above, but the pregnancy was assessed without performing ovarian US. There was no difference $(p>0.05)$ in pregnancy rate between treatments, BE $(55 \%)$ or $\mathrm{GnRH}(41 \%)$, but the follicular diameter was significantly higher $(p<0.05)$ in pregnant cows treated with EB $(10.7 \mathrm{~mm}$ vs. $8.5 \mathrm{~mm})$ and in cows treated with $\mathrm{GnRH}$ there was no difference $(p>0.05)$ between pregnant and no pregnant cows $(11.6 \mathrm{~mm}$ vs. $10.2 \mathrm{~mm}$ ). We concluded the use of $\mathrm{GnRH}$ on D0 did not improve the pregnancy rate in lactating beef cows and follicular diameter was greater $(p<0.05)$ in pregnant cows compared to non-pregnant only in G-BE.
\end{abstract}

Keywords: FTAl; post-partum; progesterone; zebu cattle.. 


\section{INTRODUÇÃO}

A pecuária de corte no Brasil caracteriza-se como uma atividade de produtividade reduzida em conseqüência, entre outros fatores, do baixo desempenho reprodutivo dos rebanhos, forçando o pecuarista a buscar melhores índices (RESTLE et al., 2002). Desta forma, elevados índices de produção, associados à alta eficiência reprodutiva, devem ser metas que norteiem os técnicos e criadores a alcançarem melhor produtividade e satisfatório retorno econômico na atividade (BARUSELLI; MARQUES, 2002).

A alta taxa de anestro, associada à baixa eficiência na detecção de cios entre as vacas que estão ciclando, resulta em uma baixa taxa de serviço. O prolongamento do período de anestro pós-parto leva a perdas econômicas (YAVAS; WALTON, 2000) por aumentar o intervalo partoconcepção e, conseqüentemente, comprometer a eficiência reprodutiva de um rebanho bovino impedindo que se atinja a meta de um parto/vaca/ano (RESTLE et al., 2002).

Assim, os protocolos hormonais de indução do estro servem como ferramentas para aumentar os índices reprodutivos. Dentre as principais vantagens destacam-se a possibilidade de viabilizar a inseminação artificial de vacas em lactação, reduzir o intervalo parto/concepção, concentrar os partos, antecipar a prenhez na temporada de monta, padronizar os lotes de bezerros e aumentar a eficiência no índice de desmame (peso ao desmame e número de animais desmamados), refletindo diretamente na racionalização da mão-de-obra e no custo/benefício da atividade (BARTOLOMEU et al., 2003; GONÇALVES et al., 2004).

Entre os protocolos para sincronização da ovulação e Inseminação Artificial em Tempo Fixo (IATF), os mais utilizados, principalmente para animais em anestro pós-parto e com boa condição corporal, são aqueles que associam uma fonte de progestágeno à aplicação de estrógenos e/ou outros hormônios (BÓ; BARUSELLI; MARTINEZ, 2003; BARUSELLI; REIS; MARQUES, 2004).

Dentre as alternativas de protocolos hormonais, a prostaglandina $(P G F 2 \alpha)$ e o benzoato de estradiol (BE) têm sido utilizados com freqüência, porém combinados a outros hormônios, tais como progesterona, seus análogos, e Hormônio Liberador de Gonadotrofina (GnRH) (MARTINEZ; BÓ; MAPLETOFT, 2004), o que aumenta os custos desses protocolos. O tratamento com benzoato de estradiol promove a liberação de um pico de Hormônio Luteinizante (LH), dentro de um intervalo de 16 a 30h (LAMMOGLIA et al., 1998). A administração de $\mathrm{GnRH}$ induz um pico de LH que se inicia logo após sua aplicação (em torno de 15 minutos).

Neste trabalho, objetivou-se avaliar a taxa de prenhez e a influência do diâmetro folicular após utilização de $\mathrm{GnRH}$ ou $\mathrm{BE}$ no dia da inserção do implante de progesterona (D0) em vacas de corte em lactação.

\section{MATERIAL E MÉTODOS}

Foram realizados 2 experimentos: Experimento 1 (Exp. 1) com exame ultrassonográfico no dia da retirada do CIDR (Controlled Internal Drug Release) (D9) para avaliar o diâmetro folicular e no experimento 2 (Exp. 2) foi avaliada a taxa de prenhez após IATF utilizando o mesmo protocolo hormonal do Exp. 1, no entanto sem avaliação do diâmetro folicular.

O Exp. 1 foi realizado em Novembro de 2008, na fazenda Santa Cecília no município de Tarabai - SP utilizando 61 vacas da raça Nelore, com idade média para as primíparas de 36 meses e para as multíparas de 60 meses, com 34 a 90 dias pós-parto e condição corporal (CC) média de 3 (escala de 1 a 5), segundo o método de Lowman et al. (1976), mantidas em pastagens de Brachiaria brizantha e sal mineralizado ad libitum. 
As vacas foram divididas aleatoriamente em dois grupos: G-BE $(n=32)$ e G-GnRH $(n=29)$. Em dia aleatório do ciclo estral (D0), os animais receberam um dispositivo intravaginal $\left(\mathrm{CIDR}^{\circledR}\right.$, Pfizer Saúde Animal, Brasil) contendo $1,9 \mathrm{~g}$ de progesterona e uma injeção de $2 \mathrm{mg}$ de benzoato de estradiol (Estrogin ${ }^{\circledR} ; 1 \mathrm{mg} / \mathrm{mL}$, Farmavet, Brasil) (G-BE) ou $0,01 \mathrm{mg}$ de $\mathrm{GnRH}$ (Conceptal ${ }^{\circledR}$; 0,04mg/10mL, Pfizer Saúde Animal, Brasil) (GGnRH), ambos por via Intra Muscular (IM). Nove dias depois (D9), o dispositivo intravaginal foi retirado e os animais foram tratados com $12,5 \mathrm{mg}$ de prostaglandina (PGF2 $\alpha$, Pfizer Saúde Animal, Brasil) via IM (Lutalyse $®, 5 \mathrm{mg} / \mathrm{mL}$ ), 0,5 mL de cipionato de estradiol (E.C.P.®, $2 \mathrm{mg} / \mathrm{mL}$, Pfizer Saúde Animal, Brasil) e feita a remoção temporária dos bezerros (RTB). Após 48 horas, ou seja, no dia 11 (D11) foi realizada a IATF, e as vacas foram inseminadas com sêmen de apenas um touro da raça Nelore, por inseminador experimente e a seguir os bezerros foram devolvidos às mães. No dia da retirada do CIDR (D9) foi realizada ultrassonografia (US) para medir o folículo dominante (FD) presente no ovário, utilizando aparelho de ultrassom (modelo HS-2000VET, Honda, Japão) com transdutor trans-retal de $5 \mathrm{MHz}$.

Após 30 dias, no mês de Dezembro de 2008, foi realizado o Exp. 2 utilizando 50 vacas da raça Nelore, com idade média de 72 meses, com 35 dias pós-parto e CC de 3 (LOWMAN et al., 1976) mantidas em pastagens de Brachiaria decumbens com suplementação mineralizada ad libitum. Inicialmente foram dividas aleatoriamente em dois grupos: G-BE $(n=22)$ e G-GnRH $(n=28)$, sendo o protocolo de sincronização para a IATF o mesmo descrito anteriormente (Exp.1), entretanto, nesse experimento não foi realizada a ultrassonografia no D9. As vacas foram inseminadas com sêmen de apenas 1 touro da raça Nelore. Em torno de 30 dias após foi realizado US para diagnóstico de prenhez em todos os grupos.

Para analisar estatisticamente a taxa de prenhez entre os grupos foi utilizado o teste Quiquadrado; para o diâmetro folicular em relação à taxa de prenhez foi utilizado o teste T de Student. As diferenças foram consideradas significativas ao nível de $5 \%$.

\section{RESULTADOS E DISCUSSÃO}

Ao avaliar estatisticamente os experimentos (1 e 2) a porcentagem de prenhez não variou entre os grupos $(p>0,05)$ e foi de $52 \%$ e $42 \%$, respectivamente nos grupos G-BE e G-GnRH (Tabela 1).

Tabela 1. Número de vacas e porcentagem de prenhez após protocolo de IATF utilizando BE ou GnRH no dia zero para indução de nova onda folicular em vacas de corte no pós-parto

\begin{tabular}{lll}
\hline & $\begin{array}{l}\text { BE } \\
(\mathbf{n}=54)\end{array}$ & $\begin{array}{l}\text { GnRH } \\
(\mathbf{n}=57)\end{array}$ \\
\hline Taxa de & $52 \%$ & $42 \%$ \\
Prenhez* & $(28 / 54)$ & $(24 / 57)$ \\
\hline *Não houve diferença & significativa \\
entre os grupos pelo & Teste Qui- \\
quadrado $(p>0,05)$. &
\end{tabular}

No grupo G-BE foi utilizado o protocolo de IATF à base de progesterona associada ao estradiol no dia zero (D0) visando a redução das concentrações de LH e Hormônio Folículo Estimulante $(\mathrm{FSH})$, causando atresia folicular e início de uma nova onda folicular, aproximadamente cinco dias após o tratamento (BÓ et al., 1995). Por outro lado, no grupo G$\mathrm{GnRH}$ testamos o uso do GnRH no D0 com o intuito de antecipar a indução da nova onda folicular, por ovulação ou acelerando a atresia folicular, a qual emerge em torno de dois dias após a aplicação do GnRH (BARROS et al., 2000). 
Também esperávamos induzir ciclicidade nos animais que apresentassem ovulação com o $\mathrm{GnRH}$, no entanto apenas $20 \%$ das vacas ovularam após a aplicação deste fármaco. Essa taxa está próximo dos obtidos por Barros et al., (2000) com 33,3\% de ovulação após aplicação de $\mathrm{GnRH}$ em vacas da raça Nelore em estádio aleatório do ciclo estral, demonstrando grande variação quando comparada à descrita por Pursley, Mee e Wiltbank (1995) em vacas de leite (90\%).

Por se tratar de fêmeas no pós-parto, a hipótese inicial era que com a indução precoce da onda folicular, no momento da retirada do implante os folículos estariam com maior diâmetro, consequentemente resultaria em melhor taxa de ovulação e maior taxa de prenhez. Essa hipótese foi em parte comprovada, pois houve aumento no diâmetro folicular no G-GnRH, no entanto, não houve diferença significativa $(p>0,05)$ na taxa de prenhez entre os grupos (Tabela 1).

Embora não houve diferença significativa, o grupo BE teve taxa de prenhez $10 \%$ maior. Desta forma, observa-se que o uso do GnRH torna-se inviável, já que é um fármaco de custo superior ao $\mathrm{BE}$. Ao contrário do que se nota em vacas de leite, os sistemas para IATF que utilizam o $\mathrm{GnRH}$ como indutor da ovulação ainda não estão adequados para serem utilizados em vacas de corte em lactação, pois produzem resultados variados e normalmente com baixas taxas de prenhez (STEVENSON et al., 2000; FERNANDES et al., 2001; BARUSELLI; MARQUES, 2002).
Dados de Fernandes et al. (2001), Baruselli e Marques (2002), Kasimanickam et al., (2006), utilizando o protocolo Ovsynch em vacas de corte em lactação, demonstraram taxas de prenhez que oscilaram entre 25 e $67 \%$. De acordo com os autores supracitados, estes resultados variam de acordo com a porcentagem de animais acíclicos no rebanho, as condições ambientais e nutricionais em que estes animais se encontram e, também, com a associação hormonal utilizada.

Em vacas com bezerro ao pé, os efeitos supressores da amamentação na secreção de $\mathrm{GnRH} / \mathrm{LH}$ impedem a manutenção do crescimento folicular após a divergência (WILTBANK; GÜMEN; SARTORI, 2002) momento em que o folículo se torna dependente da secreção pulsátil de LH (GINTHER et al., 2001). Dessa forma, o folículo dominante entra em atresia antes de ter capacidade para produzir estradiol suficiente para induzir pico de LH (WILTBANK; GÜMEN; SARTORI, 2002).

$O$ diâmetro folicular das vacas prenhes nos grupos G-BE e G-GnRH foram 10,8 mm e 11,7 $\mathrm{mm}$; e nas não prenhes de $8,5 \mathrm{~mm}$ e $10,2 \mathrm{~mm}$, respectivamente (Tabela 2). No G-BE o diâmetro folicular nas vacas prenhes $(10,8 \mathrm{~mm})$ foi significativamente maior $(p<0,05)$ que nas vacas não prenhes $(8,5 \mathrm{~mm})$. Pode-se observar que as vacas não prenhes do $\mathrm{G}-\mathrm{GnRH}$ apresentaram diâmetro apenas $0,5 \mathrm{~mm}$ menor que as prenhes do G-BE. O diâmetro folicular das vacas prenhes $(11,1 \mathrm{~mm})$ comparado ao das vacas não prenhes $(9,4 \mathrm{~mm})$, independente do tratamento, foi significativamente maior $(p<0,05)$. 
Tabela 2. Diâmetro folicular médio no dia da retirada (D9) do implante de progesterona em fêmeas prenhes e não prenhes após protocolo de IATF utilizando BE ou GnRH no D0 para indução de nova onda folicular em vacas de corte no pós-parto.

\begin{tabular}{lcc}
\hline & Prenhe & Vazia \\
\hline BE $(\mathbf{n}=\mathbf{3 2})$ & $10,76 \pm 1,75 \mathrm{aA}$ & $8,53 \pm 2,45 \mathrm{bA}$ \\
$\mathbf{G n R H}(\mathbf{n = 2 9})$ & $11,67 \pm 2,48 \mathrm{aA}$ & $10,21 \pm 2,87 \mathrm{aB}$ \\
Total $(\mathbf{n = 6 1 )}$ & $11,14 \pm 2,09 \mathrm{a}$ & $9,45 \pm 2,78 \mathrm{~b}$ \\
\hline \multicolumn{2}{l}{ Letras minúsculas diferem entre si na linha e maiúsculas diferem entre si na coluna pelo Teste T de Student } \\
$(\mathrm{p}<0,05)$.
\end{tabular}

No D9, independente da prenhez, as vacas tratadas com BE apresentaram diâmetro de 9,75 $\pm 2,34 \mathrm{~mm}$ aproximadamente, já no grupo GGnRH diâmetro de 10,81 $\pm 2,77$ mm. Embora não significativo ( $p>0,05)$, a aplicação de $\mathrm{GnRH}$ resultou em folículos com pelo menos $1 \mathrm{~mm}$ a mais que o grupo BE. Sabe-se que o diâmetro ovulatório do folículo dominante (FD) em vacas da raça Nelore é em média de $11 \mathrm{~mm}$ (FIGUEIREDO et al., 1997). Portanto, a intenção era de incrementar o diâmetro folicular, e melhorar a ovulação no final do tratamento de IATF, mas no presente experimento as vacas não prenhes do G-GnRH apresentaram diâmetro semelhante ao observado nas vacas prenhes do grupo G-BE (10,2mm vs 10,7mm).

Outros autores estudaram a relação entre o diâmetro folicular e a capacidade ovulatória e observaram que novilhas de corte européia ovulam folículos a partir de $9 \mathrm{~mm}$ (MARTINEZ et al., 1999), vacas da raça Holandesa a partir de 10 mm (SARTORI et al., 2001) e novilhas da raça Nelore a partir de $7 \mathrm{~mm}$ (GIMENES et al., 2008). Foi observado em todos esses trabalhos que a capacidade ovulatória aumenta em folículos com maior diâmetro.

Provavelmente, a baixa taxa de prenhez no G-GnRH foi resultante de um período maior de crescimento folicular e, quando foi induzido o pico de LH, a maioria dos folículos se encontrava em fases não responsivas à ovulação, ou seja, fase estática e início de regressão. Segundo Barros et al. (2000), a indução da onda está em torno de dois dias após a aplicação do $\mathrm{GnRH}$ e em torno de cinco dias com a aplicação de BE (BÓ et al., 1995). Desta forma, o FD no grupo GnRH tinha três dias a mais de crescimento no momento da retirada do implante.

Em outro estudo, em novilhas Holandesas, observou-se $100 \%$ de ovulação em folículos em fase de crescimento ( $3,8 \pm 0,2$ dias após o estro), $33 \%$ na fase estática $(7,4 \pm 0,5$ dias) e ausência de ovulação na fase de regressão $(12,7 \pm 0,5$ dias, SILCOX; POWELL; KISER, 1993).

O mesmo foi observado por Martinez et al. (1999) ao induzir ovulação com LH ou GnRH, em diferentes fases do desenvolvimento folicular: D3 (fase de crescimento), D6 (estática inicial) ou D9 (estática tardia) em novilhas Bos taurus. Neste trabalho, as novilhas tratadas com $\mathrm{LH}$ responderam com $67 \%, 100 \%$ e $67 \%$ de ovulações, enquanto aquelas tratadas com $\mathrm{GnRH}$ apresentaram $89 \%$, 56\% e $22 \%$ de ovulações, para os dias 3, 6 e 9, respectivamente. Portanto, a fase do desenvolvimento folicular exerce forte influência na capacidade ovulatória e consequentemente no sucesso dos protocolos de manipulação hormonal do ciclo estral.

\section{CONCLUSÃO}

Os resultados obtidos indicam que o uso de GnRH no D0 não melhora a taxa de prenhez em vacas de corte no pós-parto. 
O diâmetro folicular no dia da IATF foi maior nas vacas prenhes quando comparado as não prenhes somente no G-BE.

\section{COMITÊ DE ÉTICA E BIOSSEGURANÇA}

A pesquisa foi aprovada pelo Comitê de Ética da instituição de origem, protocolada sob o

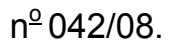

\section{AGRADECIMENTOS}

Os autores agradecem à Pfizer Saúde Animal e à Fazenda Santa Cecília.

\section{REFERÊNCIAS}

BARROS, C. M.; MOREIRA, M.B.; FIGUEIREDO, R. A.; TEIXEIRA, A. B.; TRINCA, L. A. Synchronization of ovulation in beef cows (Bos indicus) using GnRH, PGF2 $\alpha$ and estradiol benzoate. Theriogenology, v. 15, p. 1121-34, 2000. http://dx.doi.org/10.1016/S0093691X(00)00257-0.

BARTOLOMEU, C. C.; DEL REI, A. J.; OLIVEIRA, M. A. L.; LIMA, P. F.; SILVA, J. E. Inseminação artificial em tempo fixo de vacas leiteiras mestiças Holando-Zebu no pós-parto com emprego de CIDR reutilizado. Revista Brasileira de Reprodução Animal, v. 27, n. 3, p. 426-427, 2003.

BARUSELLI, P. S.; REIS, E. L.; MARQUES, M. O. Inseminação artificial em tempo fixo em bovinos de corte. In: SIMPÓSIO INTERNACIONAL DE REPRODUÇÃOANIMAL APLICADA, 1., 2004, Londrina. Anais... Londrina: [s.n.], 2004. p. 155-165.

BARUSELLI, P. S.; MARQUES, M. O. Programas de sincronização da ovulação em gado de corte. SIMPOSIO DEREPRODUÇÃO BOVINA, 1., 2002, Porto Alegre. Anais... Porto Alegre: [s.I.], 2002. p. 32-35.
BÓ, G. A.; BARUSELLI, P. S.; MARTINEZ, M. F.

Pattern and manipulation of follicular

development in Bos Indicus cattle. Animal

Reproduction Science, v. 78, p. 307-326, 2003. http://dx.doi.org/10.1016/S0378-4320(03)00097-6. BO, G. A.; ADAMS, G. P.; CACCIA, M.; MARTINEZ, M.; PIERSON, R. A.; MAPLETOFT, R. J. Ovarian follicular wave emergence after treatment with progesterone and estradiol in cattle. Animal Reproduction Science, v. 39, p. 193-204, 1995. http://dx.doi.org/10.1016/03784320(95)01389-H.

FERNANDES, P.; TEIXEIRA, A. B.; CROCCI, A. J.; BARROS, C. M. Timed artificial insemination in beef cattle using GnRH agonist PGF2alpha and estradiol benzoate. Theriogenology, v. 55, p. 1521-1532, 2001.

http://dx.doi.org/10.1016/S0093-691X(01)00499$\mathrm{X}$.

FIGUEIREDO, R. A.; BARROS, C. M.;

PINHEIRO, O. L.; SOLAR, J. M. Ovarian follicular dynamics in Nelore breed (Bos indicus) cattle.

Theriogenology, v. 47, p. 1489-1505, 1997. http://dx.doi.org/10.1016/S0093-691X(97)001568.

GIMENES, L. U.; SÁ FILHO, M. F.; CARVALHO, N. A.; TORRES-JÚNIOR, J. R.; SOUZA, A. H.; MADUREIRA, E. H.; TRINCA, L. A.; SARTORELLI, E. S.; BARROS, C. M. .; CARVALHO, J. B.; MAPLETOFT, R. J.; BARUSELLI, P. S. Follicle deviation and ovulatory capacity in Bos indicus heifers.

Theriogenology, v. 15, p. 852, 2008. http://dx.doi.org/10.1016/j.theriogenology.2008.01. 001.

GINTHER, O. J.; BEG, M. A.; BERGFELT, D. R.; DONADEU, F. X.; KOT, K. Follicle selection in monovular species. Biology of Reproduction, v. 65 , p. 638-647, 2001.

http://dx.doi.org/10.1095/biolreprod65.3.638. GONÇALVES, P. B. D.; OLIVEIRA, J. F. C.; 
SILVEIRA, R. S. FERREIRA, R. Anestro pós parto em vacas de corte. In: SIMPÓSIO INTERNACIONAL DE REPRODUÇÃO ANIMAL APLICADA, 1., 2004, Londrina. Anais... São Paulo: Faculdade de Medicina Veterinária e Zootecnia, Universidade de São Paulo, p.105116, 2004.

KASIMANICKAM, R.; COLLINS, J. C. ; WUENSCHELL, J.; CURRIN, J. C.; HALL, J. B.; WHITTIER, D. W. Effect of timing of prostaglandin administration, controlled internal drug release removal and gonadotropin releasing hormone administration on pregnancy rate in fixed-time Al protocols in crossbred Angus cows. Theriogenology, v. 66, p. 166-172, 2006. http://dx.doi.org/10.1016/j.theriogenology.2005.10. 019.

LAMMOGLIA, M. A.; SHORT, E. R.; BELLOWS, M. D.; BELLOWS, R. A.; MACNEIL, M. D.; HAFS, H. D. Induced and synchronized estrus in cattle: dose titration of estradiol benzoate in peripubertal heifers and postpartum cows after treatment with an intravaginal progesterone-releasing insert and prostaglandin F2a. Journal of Animal Science, v. 76, p. $1662-1670,1998$.

MARTINEZ, M. F.; BÓ, G. A.; MAPLETOFT, R. J. Synchronization of follicular wave emergence and ovulation for reproductive biotechnologies.

Biotecnologia da Reprodução em Bovinos. In: SIMPÓSIO INTERNACIONAL DE REPRODUÇÃO ANIMAL APLICADA, 1., 2004, Londrina. Anais... Londrina: [s.n.], 2004. p. 2655.

MARTINEZ, M. F.; ADAMS, G. P.; BERGFELT, D. R.; KASTELIC, J. P; MAPLETOFT, R. J. Effect of $\mathrm{LH}$ or $\mathrm{GnRH}$ on the dominant follicle of the first follicular wave in beef heifers. Animal

Reproduction Science, v. 57, p. 23-33, 1999. http://dx.doi.org/10.1016/S0378-4320(99)00057-3.

PURSLEY, J. R.; MEE, M. O.; WILTBANK, M. C. Synchronization of ovulation in dairy cows using
PGF2 and GnRH. Theriogenology, v. 44, p. 915-23, 1995. http://dx.doi.org/10.1016/0093691X(95)00279-H.

RESTLE, J.; NEUMANN, M.; BRONDANI, I. L.; ALVES FILHO, D. C.; SILVA, J. H. S.; GONÇALVES, J. M.; KUSS, F. Produção do superprecoce a partir de bezerros desmamados aos 72 ou 210 dias de idade. Revista da

Sociedade Brasileira de Zootecnia, v. 31, n. 4, p. 1803-1813, 2002.

http://dx.doi.org/10.1590/S1516-

35982002000700016.

SARTORI, R.; FRICKE, P. M.; FERREIRA, J. C. P.; GINTHER, O. J.; WILTBANK, M. C. Follicular deviation and acquisition of ovulatory capacity in bovine follicles. Biology of Reproduction, v. 65, p. 1403-1409, 2001. http://dx.doi.org/10.1095/biolreprod65.5.1403. SILCOX, R. W.; POWELL, K. L.; KISER, T. E. Ability of dominant follicles (DF) to respond to exogenous $\mathrm{GnRH}$ administration is dependent on their stage of development. Journal of Animal Science, v. 71, p. 219, 1993.

STEVENSON, J. C. ; THOMPSON, K. E.; FORBES, W. L. LAMB, G. C.; GRIEGER, D. M.; CORAH, L. R. Synchronizing estrus and (or) ovulation in beef cows after combinations of $\mathrm{GnRH}$, norgestomet, and prostaglandin F2a with or without timed insemination. Journal of Animal Science, v. 78, p. 1747-1758, 2000.

YAVAS, Y.; WALTON J. S. Postpartum acyclicity in suckled beef cows: a review. Theriogenology, v. 54 , p. 25-55, 2000.

http://dx.doi.org/10.1016/S0093-691X(00)00323$\mathrm{X}$.

WILTBANK, M. C.; GÜMEN, A.; SARTORI, R. Physiological classification of anovulatory conditions in cattle. Theriogenology, v. 57, p. 21-52, 2002. http://dx.doi.org/10.1016/S0093691X(01)00656-2. 